Series, 68, No. 126, 252 (1972).

7) Jonke, A. A., G. J. Vogel, E. L. Carls et al.: Chem. Eng. Prog., Symp. Series, 68, No. 126241 (1972).

8) Muzio, L. J., J. K. Arand and D. P. Texeira: 16th Symp. on Combustion, p. 199 (1976).

9) Nagashima, I., N. Yamamoto, T. Fujikawa, T. Furusawa and D. Kunii: Kagaku Kogaku Ronbunshu, 3, 236 (1977).
10) Pereira, F. J., J. M. Beér, B. Gibbs and A. B. Hedley: 15th International Symp. on Combustion, Tokyo, 1149 (1974).

(Presented at the 10th (at Nagoya, Oct. 15, 1976) and the 11th (at Tokyo, Oct. 6, 1977) Autumn Meeting of The Soc. of Chem. Engrs., Japan and at the 2nd Pacific Chem. Eng. Congress at Denver, Colorado, Aug. 30, 1977.)

\title{
COMBINED PROCESS OF REACTION, EXTRACTION AND DISTILLATION IN A MULTISTAGE COLUMN
}

\author{
Shigeo GOTO and MaSakazU MATSUBARA \\ Department of Chemical Engineering, \\ Nagoya University, Nagoya 464
}

\begin{abstract}
Reactive-extractive distillation in a multistage column is numerically investigated to improve the yield of desired intermediate product in a complex reaction combining reversible and consecutive reactions which are of the first order. On the assumption of phase equilibrium, mass balance equations are formulated in terms of a phase-splitting parameter and solved by use of the modified relaxation method.

The optimal value of each decision variable is determined for tentatively chosen parameter values. Then the effect of each parameter on yield is evaluated for fixed values of the remaining parameters. The proposed combined process may have advantages over extractive reaction, reactive distillation and reaction without simultaneous separation singly if the system parameters can be chosen favorably.
\end{abstract}

\section{Introduction}

Separate equipment used to be provided for each chemical reaction and each physical unit operation in conventional chemical process design. However, if the desired products are intermediates of a complex reaction which may be rapidly converted into wasteful final products, process performance can be improved by selective removal of the desired products from the reaction system. In other words, simultaneous operation which is a combination of chemical reaction and physical unit operation in the same equipment may be more profitable than successive operations in separate equipment. For example, it is shown that in esterification a reactive distillation ${ }^{4,5)}$ and an extractive reaction ${ }^{6)}$ can achieve conversions higher than that for chemical equilibrium in a conventional reactor.

In the previous paper ${ }^{2}$, some basic considerations were given for assuring the advantage of the combined process where three operations of reaction, extraction and distillation were simultaneously carried out in a

Received November 24, 1977. Correspondence concerning this article should be addressed to S. Goto. single stirred tank. In this paper, a multistage distillation column accompanied by reaction and extraction is studied to clarify the effect of each parameter on the yield of the intermediate product in a complex reaction. This combined process, referred to as reactive-extractive distillation, is compared with three other processes, namely reactive distillation, extractive reaction and reaction without simultaneous separation.

\section{System Description}

Consider a distillation column with a separator shown in Fig. 1. The plate stages are numbered from 1 to $n-1$ starting at the top plate. The condenser, reboiler and separator are numbered $0, n$ and $n+1$, respectively. The raw material $\mathrm{A}$ is fed to the $f \mathrm{th}$ stage and a complex reaction combining reversible and consecutive reactions

$$
\mathrm{A} \underset{k_{R}}{\stackrel{k}{\rightleftarrows}} \mathrm{R} \stackrel{k_{C}}{\longrightarrow} \mathrm{S}
$$

takes place in each stage, where $\mathrm{R}$ is the desired product and $S$ the wasteful product. An appropriate solvent which can selectively extract $R$ from the 
mixture of $\mathrm{A}, \mathrm{R}$ and $\mathrm{S}$ is fed to the sth stage. Thus, in eax phase containing the greater part of A and the extractive phase containing the greater part of $R$. At the separator, the extractive phase being rich in the desired product is to be taken out of the column, while the reactive phase being rich in the unreacted raw material is to be recycled to the reboiler. However, it might be necessary for satisfying the total mass balance either to take out a portion of the reactive phase or to feed back a portion of the extractive phase.

To keep the calculation as simple as possible the following assumptions are made:

1. The solvent is involatile and also insoluble in the reactive phase. Therefore, the liquid phase in the zeroth to the $(s-1)$ th stage does not include the extractive phase.

2. All the reactions are of the first order. The reaction rate constants are constant throughout the column and the ratio of rate constants in the reactive and the extractive phase is identical for the three reactions $\mathrm{A} \rightarrow S, \mathrm{R} \rightarrow \mathrm{A}$ and $\mathrm{R} \rightarrow \mathrm{S}$. The progress of reaction in the condenser and separator is negligible.

3. The rates of mass transfer between the vapor phase and the reactive phase and those between the reactive phase and the extractive phase are so much higher than the rates of chemical reactions that each stage can be regarded as always in phase equilibrium. Further, the relative volatility between components and the distribution coefficients of each component are constant throughout the column.

4. Each separate phase in each stage is instantaneously well mixed. The reactive and the extractive phase in the $s$-th to the $n$-th stage are always uniformly dispersed in each other.

5. Vapor holdup between stages is negligible and consequently the progress of chemical reaction in the vapor phase is also negligible.

6. Vapor rate is constant throughout the column.

7. Total liquid holdup in each stage is held constant.

8. The raw material feed, the solvent feed, the reflux and the recycle stream from the separator enter the corresponding stages as liquid at the temperature within the destination stage. The column is operating adiabatically.

For convenience four components, A, R, S and the solvent, are numbered 1,2,3 and 4, respectively, and the compositions of the $i$-th component within the $j$-th stage in the reactive phase, the extractive phase and the vapor phase are denoted by $x_{i, j}, x_{i, j}^{\prime}$ and $y_{i, j}$, respectively. Since $x_{4, j}$ and $y_{4, j}$ are equal to zero by assumption 1, one has

$$
\sum_{i=1}^{3} x_{i j}=1, \sum_{i=1}^{4} x_{i, j}^{\prime}=1 \text { and } \sum_{i=1}^{3} y_{i, j}=1
$$

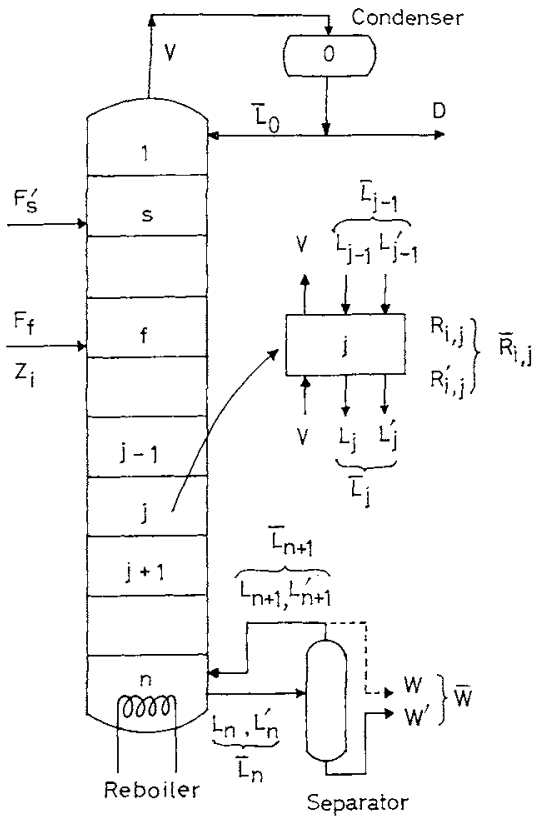

Fig. 1 Schematic of distillation column accompanied by reaction and extraction

By assumption $3 x_{i, j}, x_{i, j}^{\prime}$ and $y_{i, j}$ are related as

$$
\begin{aligned}
y_{i, j} & =K_{i, j}^{*} x_{i, j}=\alpha_{i} K_{1, j}^{*} x_{i, j} \\
i & =1,2,3 ; j=1, \cdots, n \\
x_{i, j}^{r} & =m_{i} x_{i, j} \\
i & =1,2,3 ; j=s, \cdots, n+1
\end{aligned}
$$

where the relative volatility $\alpha_{i}\left(=K_{i, j}^{*} / K_{1, j}^{*}\right)$ and the distiribution coefficient $m_{i}$ are constant and independent of stage number.

Holdups, flow rates and time are divided by $\bar{H}_{t}$ $\left(=\sum_{j=1}^{n} \bar{H}_{j}\right), F_{f}$ and $\bar{H}_{i} / F_{f}$ to yield normalized holdups, normalized flow rates and normalized time, respectively. Thus,

$$
\begin{aligned}
& \bar{h}_{j}=\bar{H}_{j} / \bar{H}_{t}, h_{i}=H_{j} / \bar{H}_{t}, h_{j}^{\prime}=H_{j}^{\prime} / \bar{H}_{t} \\
& f_{s}=F_{s}^{\prime} / F_{f}, v=V / F_{f}, d=D / F_{f}, \bar{l}_{j}=\bar{L}_{j} / F_{f}, l_{j}=L_{j} / F_{f}, \\
& l_{j}^{\prime}=L_{j}^{\prime} / F_{f}, \bar{w}=\bar{W} / F_{f}, w=W / F_{f}, w^{\prime}=W^{\prime} / F_{f} \\
& \theta=t /\left(\bar{H}_{t} / F_{f}\right)
\end{aligned}
$$

where notations for liquid holdups or liquid flow rates are distinguished into three classes: those with bars for the whole liquid phase, those without markings for the reactive phase and those with primes for the extractive phase. Evidently, the following relations hold between these quantities:

$$
\begin{aligned}
& \bar{h}_{j}=h_{j}+h_{j}^{\prime} \quad j=0,1, \cdots, n \\
& \bar{l}_{j}=l_{j}+l_{j}^{\prime} \quad j=0,1, \cdots, n+1 \\
& \bar{w}=w+w^{\prime}
\end{aligned}
$$

The average composition of each component in the liquid phase leaving each stage can be written as

$$
\begin{aligned}
\bar{x}_{i, j} & =\gamma_{j} x_{i, j}+\left(1-\gamma_{j}\right) x_{i, j}^{\prime} \\
i & =1, \cdots, 4 ; \quad j=0, \cdots, n+1
\end{aligned}
$$




$$
\begin{gathered}
\bar{x}_{i, w}=\gamma_{w} x_{i, n+1}+\left(1-\gamma_{w}\right) x_{i, n+1}^{\prime} \\
i=1, \cdots, 4
\end{gathered}
$$

by use of the phase-splitting parameter $\gamma_{j}$ introduced by Block and Hegner ${ }^{1)}$ where

$$
\begin{aligned}
& \gamma_{j}=l_{i} / \bar{l}_{j} \quad j=0,1, \cdots, n+1 \\
& \gamma_{w}=w / \bar{w}
\end{aligned}
$$

The separator has two leaving streams. One is recycled to the reboiler with composition $\tilde{x}_{i, n+1}$ and flow rate $\bar{l}_{n+1}$ and the other is taken out of the column with composition $\bar{x}_{i, w}$ and flow rate $\bar{w}$. Since the zeroth to the $(s-1)$ th stage do not include the extractive phase, it is evident that

$$
\gamma_{j}=1 \quad j=0, \cdots, s-1
$$

The reaction rates in the reactive phase are

$$
\begin{array}{ll}
R_{1, j}=-k C_{t} x_{1, j}+k_{R} C_{t} x_{2, j} & j=1, \cdots, n \\
R_{2, j}=k C_{t} x_{1, j}-\left(k_{R}+k_{C}\right) C_{t} x_{2, j} & j=1, \cdots, n \\
R_{3, j}=k_{C} C_{t} x_{2, j} & j=1, \cdots, n \\
R_{4, j}=0 & j=1, \cdots, n
\end{array}
$$

and those in the extractive phase are given by attaching primes to all the notations in Eq. (12). These reaction rates are multiplied by $\bar{H}_{t} / F_{f}$ to yield normalized reaction rates, $r_{i, j}=R_{i, j} \bar{H}_{t} / F_{f}$ and $r_{i, j}^{\prime}=R_{i, j}^{\prime} \bar{H}_{t} / F_{f}$. Since one has by assumption 4

$$
h_{j} / \bar{h}_{j}=l_{j} / \vec{l}_{j}=\gamma_{j}
$$

the average normalized reaction rates become

$$
\vec{r}_{i, j}=\gamma_{j} r_{i, j}+\left(1-\gamma_{j}\right) r_{i, j}^{\prime}
$$

Using Eqs. (3), (7), (12), (13) and (14) yields

$$
\begin{aligned}
& \bar{r}_{1, j}=\gamma_{j} D a\left\{-\left(1+k_{j} m_{1}\right) x_{1, j}+\left(1+\kappa_{j} m_{2}\right) \kappa_{R} x_{2, j}\right\} \text { (15-a) } \\
& \bar{r}_{2, j}=\gamma_{j} D a\left\{\left(1+\kappa_{j} m_{1}\right) x_{1, j}-\left(1+\kappa_{j} m_{2}\right)\left(\kappa_{R}+\kappa_{C}\right) x_{2, j}\right\}
\end{aligned}
$$

$$
\begin{aligned}
& \bar{r}_{3, j}=\gamma_{j} D a\left(1+\kappa_{j} m_{3}\right) x_{2, j} \\
& \bar{r}_{4, j}=0
\end{aligned}
$$

where

$$
\begin{aligned}
D a & =k C_{t} \bar{H}_{t} / F_{f} \\
\kappa_{j} & =k^{\prime} C_{t}^{\prime}\left(1-\gamma_{j}\right) /\left(k C_{t} \gamma_{j}\right)=\kappa\left(1-\gamma_{j}\right) / \gamma_{j}
\end{aligned}
$$

The mass balance equation of each component around each stage becomes

$$
\begin{aligned}
\bar{h}_{0} d \bar{x}_{i, 0} / d \theta= & v y_{i, 1}-\left(\bar{l}_{0}+d\right) \bar{x}_{i, 0} \\
\bar{h}_{j} d \bar{x}_{i, j} / d \theta= & \bar{l}_{j-1} \bar{x}_{i, j-1}+v y_{i, j+1}-\bar{l}_{j} \bar{x}_{i, j}-v y_{i, j} \\
& +\bar{r}_{i, j} \bar{h}_{j}+\delta_{i f} z_{i}+\delta_{j s} f_{s} \\
& j=1, \cdots, n-1 \\
\bar{h}_{n} d \bar{x}_{i, n} / d \theta= & \bar{l}_{n-1} \bar{x}_{i, n-1}+\bar{l}_{n+1} \bar{x}_{i, n+1}-\bar{l}_{n} \bar{x}_{i, n}-v y_{i, n} \\
& +\bar{r}_{i, n} \bar{h}_{n}+\delta_{n f} z_{i}+\delta_{n s} f_{s} \\
\bar{h}_{n+1} d \bar{x}_{i, n+1} / d \theta= & \bar{l}_{n} \bar{x}_{i, n}-\bar{l}_{n+1} \bar{x}_{i, n+1}-\bar{w} \bar{x}_{i, w}
\end{aligned}
$$

where $\delta_{i f}$ and $\delta_{j s}, j=1, \cdots, n$ are Kronecker's delta. By assumption 7 the total mass balance around each stage becomes

$$
\begin{aligned}
& v-\bar{l}_{0}-d=0 \\
& \bar{l}_{j-1}-\bar{l}_{j}+\delta_{i f}+\delta_{j s} f_{s}=0 \quad j=1, \cdots, n-1 \\
& \bar{l}_{n-1}+l_{n+1}-\bar{l}_{n}-v+\delta_{n f}+\delta_{n s} f_{s}=0 \\
& \bar{l}_{n}-\bar{l}_{n+1}-\bar{w}=0
\end{aligned}
$$

These mass balance equations are similar to those for two-phase distillation due to Block and Hegner ${ }^{1>}$ because of using the phase-splitting parameter.

One can easily obtain from Eq. (19)

$$
\begin{aligned}
& \bar{l}_{j}= \begin{cases}v-d & 0 \leq j<\min (f, s) \\
v-d+f_{s} & s \leq j<f \\
v-d+1 & f \leq j<s \\
v-d+f_{s}+1 & \max (f, s)<j \leq n\end{cases} \\
& \bar{l}_{n+1}=v \\
& \bar{w}=f_{s}+1-d
\end{aligned}
$$

Hence, if the values of $f_{s}, v$ and $d$ are given, one can determine the values of $\bar{l}_{j}, j=0, \cdots, n+1$ and $\bar{w}$ by use of Eq. (20).

In this work only the steady-state behavior of the above-described system is investigated. In the steady state the relations

$$
x_{i, n+1}=x_{i, n} \text { and } x_{i, n+1}^{\prime}=x_{i, n}^{\prime}
$$

hold because of assumption 2 . Since the extractive phase in the separator is taken out of the column prior to the reactive phase, the following relations hold in the steady state.

$$
\begin{array}{lll}
w^{\prime}=l_{n}^{\prime}, w=\bar{w}-l_{n}^{\prime} & \text { if } & l_{n}^{\prime}<\bar{w} \\
w^{\prime}=l_{n}^{\prime}=\bar{w}, w=0 & \text { if } & l_{n}^{\prime}=\bar{w} \\
w^{\prime}=\bar{w} . \quad w=0 & \text { if } & l_{n}^{\prime}>\bar{w}
\end{array}
$$

In the first case the reactive phase should be taken out of the column at the rate of $\bar{w}-l_{n}^{\prime}$, while in the third case the extractive phase should be recycled to the reboiler at the rate of $l_{n}^{\prime}-\bar{w}$. Therefore, in the second case the system will be in the most desirable state. In the steady state one has

$$
\bar{x}_{i, 0}=y_{i, 1}
$$

from Eqs. (18-a) and (19-a) and

$$
\bar{l}_{n+1} \bar{x}_{i, n+1}-\bar{l}_{n} \bar{x}_{i, n}=-w x_{i, n}-w^{\prime} x_{i, n}^{\prime}
$$

from Eqs. (18-d) and (21). Therefore, Eq. (18) reduces to

$$
\begin{aligned}
\bar{h}_{1} d \bar{x}_{i, 1} / d \theta= & v y_{i, 2}-\bar{l}_{1} \bar{x}_{i, 1}-d y_{i, 1}+\bar{r}_{i, 1} \bar{h}_{1} \\
& +\delta_{1 f} z_{i}+\delta_{1 s} f_{s} \\
\bar{h}_{j} d \bar{x}_{i, f} / d \theta= & \bar{l}_{j-1}+v y_{i, j+1}-\bar{l}_{j} \bar{x}_{i, j}-v y_{i, j}+\bar{r}_{i, j} \bar{h}_{j} \\
& +\delta_{i f} z_{i}+\delta_{i s} f_{s} \quad j=2, \cdots, n-1 \\
\bar{h}_{n} d \bar{x}_{i, n} / d \theta= & \bar{l}_{n-1} \bar{x}_{i, n-1}-w x_{i, n}-w^{\prime} x_{i, n}^{\prime}-v y_{i, n} \\
& +\bar{r}_{i, n} \bar{h}_{n}+\delta_{n f} z_{i}+\delta_{n s} f_{s}
\end{aligned}
$$

Of course, in the steady state the left-hand sides of Eq. (25) must be equal to zero. However, the modified relaxation method in which the dynamic equations are integrated to obtain the steady state is adopted for the 
present investigation and therefore the left-hand sides of Eq. (25) are left as they are. The right-hand sides of Eq. (25) include $\gamma_{j}, x_{i, j}$ and $y_{i, j}$ besides $\bar{x}_{i, j}$ because $\vec{r}_{i, j}$ in Eq. (15) includes $\gamma_{j}$ and $x_{i, j}$. However, one can derive from Eqs. (1), (2), (3) and (7)

$$
\begin{aligned}
& x_{i, j}=\frac{\bar{x}_{i, j}}{\gamma_{j}+\left(1-\gamma_{j}\right) m_{i}} \quad j=1, \cdots, n \\
& y_{i, j}=\frac{\alpha_{i} K_{1, j}^{*} \bar{x}_{i, j}}{\gamma_{j}+\left(1-\gamma_{j}\right) m_{i}} \quad j=1, \cdots, n \\
& \sum_{i=1}^{3} \frac{\bar{x}_{i, j}}{\gamma_{j}+\left(1-\gamma_{j}\right) m_{i}}=1 \quad j=1, \cdots, n
\end{aligned}
$$

Hence, $\gamma_{j}, x_{i, j}$ and $y_{i, j}$ can be eliminated from the righthand side of Eq. (25). Consequently, Eq. (25) can be solved with respect to $\bar{x}_{i, j}, i=1, \cdots, 4 ; j=1, \cdots, n$ if the values of $f_{s}, v, d, \bar{h}_{j}(j=1, \cdots, n)$ and all the parameters are given.

For simplifying the calculation the temperature dependency of physical and kinetic parameters is not taken into account in this investigation. Using Eqs. (1) and (2) yields

$$
K_{1, j}^{*}=1 / \sum_{i=1}^{3} \alpha_{i} x_{i, j}
$$

Therefore, if $K_{j, j}^{*}$ is known as a function of temperature, the temperature in each stage can be determined. Thus, it is not impossible to take the temperature dependency into account.

\section{Computational Procedure for Determining the Steady-State Performance}

The steady-state composition profile in the reactiveextractive distillation system described in the last section can be determined for tentatively chosen values of parameters and assumed values of decision variables. Then the yield

$$
\eta=\frac{\left(\begin{array}{l}
\text { the withdrawal rate of } \mathrm{R} \\
\text { in the extractive phase }
\end{array}\right)}{\text { the feed rate of } \mathrm{A}}=\frac{w^{\prime} x_{2, n+1}^{\prime}}{z_{1}}
$$

can be calculated. The values of decision variables which maximize the value of $\eta$ can also be sought.

Assuming that no chemical reaction proceeds within the feed line of raw material, the feed compositions were chosen as

$$
z_{1}=1, z_{2}=z_{3}=z_{4}=0
$$

The reactive-extractive distillation is successfully applicable to a set of four components having such properties that the values of $\alpha_{3}$ and $m_{2}$ are large while those of $\alpha_{2}, m_{1}$ and $\kappa$ are small. Assuming the existence of such a set, the phase equilibrium parameters and the kinetic parameters were tentatively chosen as

$$
\alpha_{2}=0.5, \alpha_{3}=2.0, m_{1}=0.1, m_{2}=10, m_{3}=1
$$

and

$$
\kappa=0.01, \kappa_{R}=9, \kappa_{C}=1
$$

respectively.

The $\bar{h}_{0}$ and $\bar{h}_{n+1}$ need not be specified because these values do not depend on the steady state. The total liquid holdups of the remaining stages are for simplicity set equal to each other as

$$
\bar{h}_{j}=1 / n \quad j=1, \cdots, n
$$

Thus, one has seven decision variables $n, f, s, v, d, f_{s}$ and $D a$. As the values of $n$ and $v$ are increased, the value of $\eta$ will also be increased with gradually decreasing rate. Economic considerations should also be taken because the increase in $n$ and $v$ causes an increase in cost. The conversion of $A$ into $R$ as well as the selective extraction of $R$ into the extractive phase could be promoted by feeding both raw material and solvent to the first stage. It is necessary for recovering the unreacted raw material to take out no top product. From these points of view the values of these decision variables were chosen as

$$
n=5, f=s=1, v=5, d=0
$$

Finally, one has only two decision variables, $f_{s}$ and $D a$ whose values are to be adjusted for maximizing the value of $\eta$. At the optimal point the equality $l_{n}^{\prime}=\bar{w}$ will be satisfied as previously conjectured.

The steady-state composition profile for fixed values of $f_{s}$ and $D a$ was calculated by use of the modified relaxation method developed by Ishikawa and Hirata $^{3}$. Starting from the initial guess of $\bar{x}_{i, j}, j=1, \cdots, n$ the set of Eq. (25) was numerically integrated until steady state was reached. The computation procedure was always numerically stable and the steady state finally reached was always independent of the initial guess.

\section{Optimization and Comparison with Alternative Processes}

The values of $f_{s}$ and $D a$ which maximize the value of $\eta$ were determined for parameter values in Eq. (32) and values of the remaining decision variables in Eq. (34). In the first step the value of $D a$ which maximizes the value of $\eta$ for each fixed value of $f_{s}$ was determined. The results indicated that the equality $l_{n}^{\prime}=\bar{w}$ was always satisfied at the optimal point as in the example shown in Fig. 2. As the value of $D a$ exceeds its optimal value, the value of $l_{n}^{\prime}$ is rapidly increased because the excess of the extractive phase must be recycled from the separator to the reboiler. In the next step the resulted maximal value of $\eta$ for each fixed value of $f_{s}$ versus the corresponding value of $D a$ was plotted (curve A) together with the value of $f_{s}$ (dotted line) as shown in Fig. 3. For comparison with alternative processes, $\eta$ versus $D a$ curves for extractive reaction using a stirred tank reactor with a phase separator (curve B), reactive distillation using a multistage distillation column with solvent feed (curve 


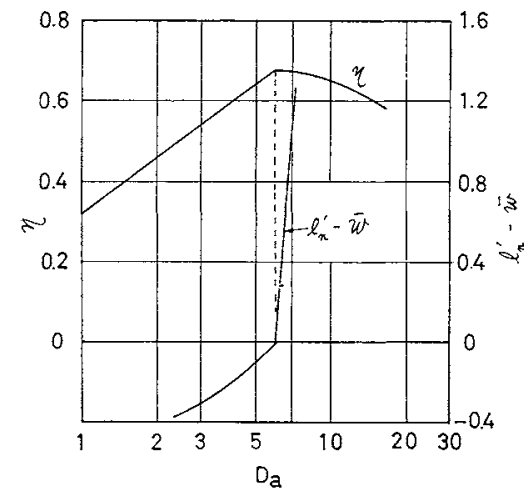

Fig. $2 \eta$ and $l_{n}^{\prime}-\bar{w}$ versus $D a$ for $f_{s}=0.1$

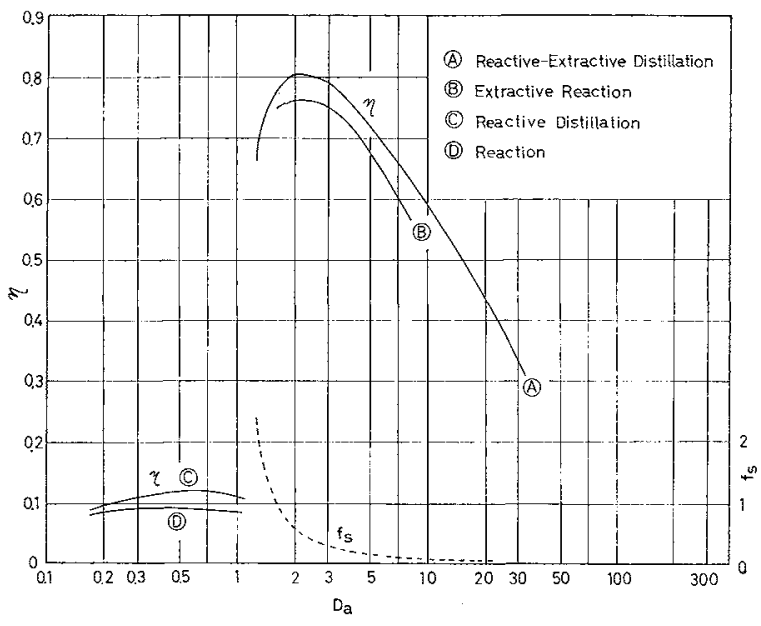

Fig. 3 Optimization of four alternative processes

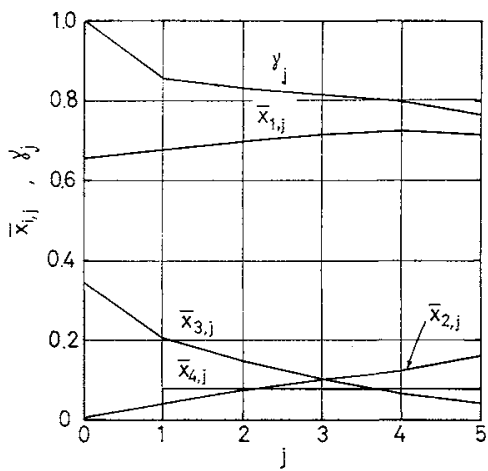

Fig. 4 Profiles of average compositions and phase-splitting parameter in the optimal steady state

C) and reaction without any simultaneous separations using a plug flow reactor (curve D) are also plotted, where values of parameters and decision variables (other than $D a$ ) are chosen equal to those included in Eqs. (31)-(34) as they are needed. The results can be summarized as follows:

Reactive-extractive distillation

$$
\eta_{\mathrm{max}}=0.805, D a=2.05, f_{s}=0.518
$$

Extractive reaction

$$
\eta_{\text {max }}=0.765, D a=2.29, f_{s}=0.715
$$

Reactive distillation

$$
\eta_{\mathrm{max}}=0.118, D a=0.565
$$

Reaction without separation

$$
\eta_{\max }=0.088, D a=0.442
$$

The advantage of reactive-extractive distillation over the remaining three processes is obvious as far as maximal attainable yield is concerned.

Figure 4 indicates the profile of the average composition $\bar{x}_{i, j}$ and the phase-splitting parameter $\gamma_{j}$ in the optimal steady state. Since the relative volatility of desired product $\alpha_{2}$ is small, its average composition $\bar{x}_{2, j}$ increases downward. Conversely, $\vec{x}_{3, j}$ increases upward because of large $\alpha_{3}$. Since the distribution coefficient of desired product $m_{2}$ is large relative to $m_{1}$ and $m_{3}$, the desired product is efficiently extracted from the reactive phase to the extractive phase and in consequence the phase-splitting factor $\gamma_{j}$ decreased downward.

\section{Effects of Changes in Decision Variables on the Yield}

The values of some decision variables in Eq. (34) were chosen on the basis of some conjectures.

In this section the validity of these conjectures is examined. To this end, the value of one parameter in Eq. (34) is varied at a time and the values of $f_{s}$ and $D a$ which maximize the value of $\eta$ are determined. Results are shown in Figs. 5-8 where the maximal attainable yield $\eta_{\max }$ and the corresponding optimal values of $f_{s}$ and $D a$ are plotted for varied values of decision variables or parameters.

Figures 5 and 6 indicate the validity of the conjecture that the increase in the total number of stages $n$ or in the vapor flow rate $v$ results in the increase in $\eta_{\text {max }}$. As seen in these figures the increase in $\eta_{\max }$ is quite slow in the region of $n$ or $v$ larger than the value chosen in Eq. (34).

Figure 7 indicates that the solvent is to be fed to the first stage.

It is observed in Fig. 8 that the value of $\eta_{\max }$ is rapidly decreased as the value of $d$ is increased. This means that the unreacted raw material is lost from the top of the column as conjectured.

\section{Conclusion}

Some basic characteristics of reactive-extractive distillation in a multistage column were clarified through numerical calculations for tentatively chosen parameter values. The results indicate that both raw material and solvent are to be fed to the top stage, that distillate is not to be wirthdrawn and that all the extractive phase and no reactive phase are to be withdrawn from the separator. Further, if a set of raw 


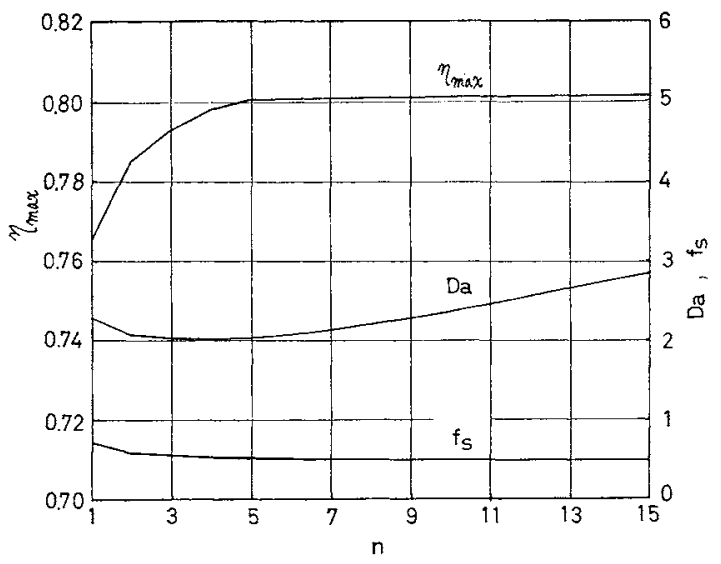

Fig. 5 Effect of change in the number of stages $n$ on $\eta_{\max }$

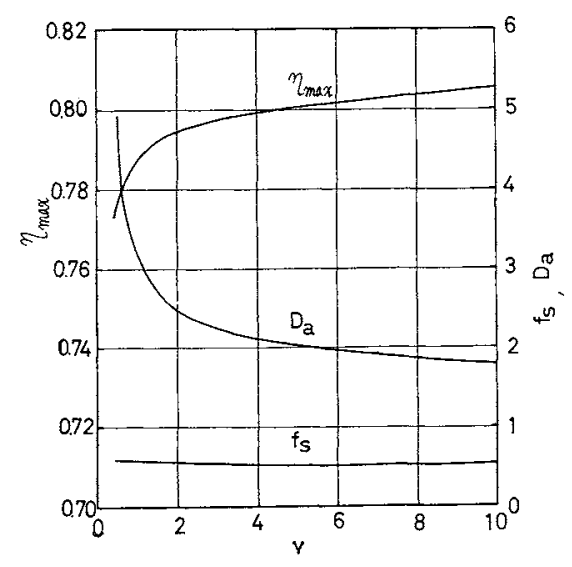

Fig. 6 Effect of change in the vapor rate $v$ on $\eta_{\max }$

material and solvent having favorable parameter values could be found, the proposed combined process may have advantages over any of extractive reaction, reactive distillation and reaction without simultaneous separation singly.

\section{Nomenclature}

$\begin{array}{ll}C_{t}, C_{t}^{\prime} & =\text { total concentration } \\ D & =\text { distillate rate } \\ D a & =k C_{t} \bar{H}_{t} / F_{f} \\ d & =D / F_{f} \\ F_{f} & =\text { feed rate of reactive phase } \\ F_{s}^{\prime} & =\text { feed rate of solvent } \\ f & =\text { stage number of raw material feed } \\ f_{s} & =F_{s}^{\prime} / F_{f} \\ \bar{H}_{j}, H_{j}, H_{j}^{\prime} & =\text { liquid holdup at } j \text { th stage } \\ \bar{H}_{t} & =\text { total liquid holdup, } \sum_{j=1}^{n_{1}} \bar{H}_{j} \\ \bar{h}_{j}, h_{j}, h_{j}^{\prime} & =\bar{H}_{j} / \bar{H}_{i}, H_{j} / \bar{H}_{i}, H_{j}^{\prime} / \bar{H}_{i} \\ K_{i, j}^{*} & =\text { equilibrium ratio } \\ k, k_{R}, k_{C} & =\text { rate constant of first order } \\ \bar{L}_{j}, L_{j}, L_{j}^{\prime} & =\text { liquid flow rate } \\ \bar{l}_{j}, l_{j}, l_{j}^{\prime} & =\bar{L}_{j} / F_{f}, L_{j} / F_{f}, L_{j}^{\prime} / F_{f} \\ m_{i} & =\text { distribution coefficient } \\ n & =\text { total number of stages }\end{array}$

$\left[\mathrm{mol} / \mathrm{cm}^{3}\right]$ $[\mathrm{mol} / \mathrm{s}]$

[-]

$$
[-]
$$$$
[-]
$$

$\left[\mathrm{cm}^{3}\right]$

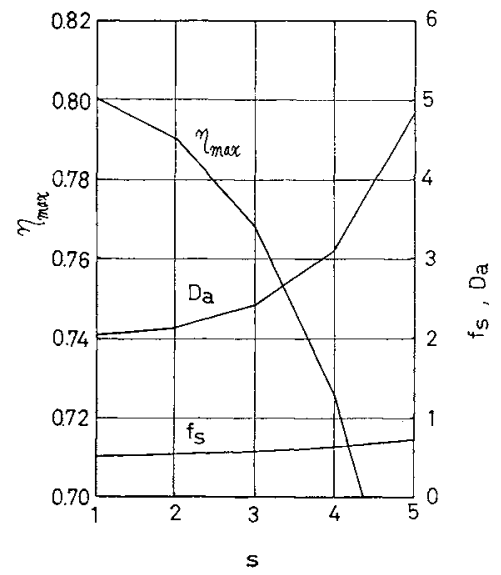

Fig. 7 Effect of change in the stage number of solvent feed $s$ on $\eta_{\max }$

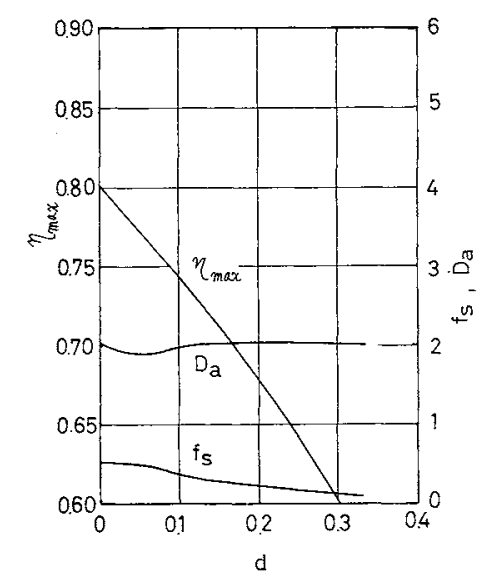

Fig. 8 Effect of change in the distillate rate $d$ on $\eta_{\max }$

$\bar{R}_{i, j}, R_{i, j}, R_{j, j}^{\prime}=$ reaction rate at $j$-th stage $\quad\left[\mathrm{mol} / \mathrm{s} \cdot \mathrm{cm}^{3}\right]$ $\bar{r}_{i, j}, r_{i, j}, r_{i, j}^{\prime}=\bar{R}_{i, j} \bar{H}_{t} / F_{f}, R_{i, j} \bar{H}_{t} / F_{f}, R_{i, j}^{\prime} \bar{H}_{t} / F_{f}$ $s \quad=$ stage number of solvent feed $\quad[-]$

$$
t \quad=\text { time [s] }
$$$$
V \quad=\text { vapor flow rate } \quad[\mathrm{mol} / \mathrm{s}]
$$

$$
v \quad=V / F_{f}
$$

$\bar{W}, W, W^{\prime}=$ flow rate withdrawn from separator [mol/s]

$$
\bar{w}, w, w^{\prime}=\dddot{W} / F_{f}, W / F_{f}, W^{\prime} / F_{f}
$$

[-] $\bar{x}_{i, j}, x_{i, j}, x_{i, j}^{\prime}=$ liquid composition, mole fraction [-]

$[\mathrm{mol} / \mathrm{s}] \quad y_{i, j} \quad=$ vapor composition, mole fraction $\quad[-]$

$[\mathrm{mol} / \mathrm{s}] \quad z_{i} \quad=$ feed composition, mole fraction $[-]$

$\alpha_{i} \quad=$ relative volatility

$[-]$

$\gamma_{j}, \gamma_{w}=$ phase-splitting parameter $\quad[-]$

$\eta \quad=$ yicld $\quad[-]$

$\theta \quad=t /\left(\bar{H}_{t} / F_{f}\right)$

$[-]$

[-]

[-]

$[-]$ 


$$
\begin{array}{ll}
- & =\text { average or total } \\
1 & =\text { component } \mathrm{A} \\
2 & =\text { component } \mathrm{R} \\
3 & =\text { component } \mathrm{S} \\
4 & =\text { solvent }
\end{array}
$$

\section{Literature Cited}

1) Block, U. and B. Hegner: AIChE J., 22, 582 (1976).

2) Goto, S. and M. Matsubara: J. Chem. Eng. Japan, 9, 338 (1976).
3) Ishikawa, T. and M. Hirata: ibid., 5, 125 (1972).

4) Nishi, Y: Kagaku Kōgaku: 35, 892 (1971).

5) Suzuki, I., H. Yagi, H. Komatsu and M. Hirata: J. Chem. Eng. Japan, 4, 26 (1971).

6) Washino, K., S. Goto and M. Matsubara: Kagaku Kögaku, 38, 869 (1974).

(Presented at the 11th Autumn Meeting of The Soc. of Chem. Engrs., Japan at Tokyo, Oct. 1977.)

\title{
SAFETY SUPERVISION OF VALVE OPERATIONS
}

\author{
EIII O'SHIMA \\ Research Laboratory of Resources Utilization, \\ Tokyo Institute of Technology, Yokohama 227
}

For the purpose of safety control of chemical plant operations, the system of supervising valve operations is considered on the premise that all the open-or-closed information for important valves can be monitored by a computer.

The algorithms consist basically of two parts: finding the particular route of a stream on specification of the starting and terminating points, and evaluating the flow state in each unit of equipment in the stream. For ease of computer processing the structure of the chemical plant is viewed as an assembly of connectors and valves as done by Rudd.

In the procedure of searching routes, redundant searching and repetition of searching in loops are avoided. Once the required route is found, the flow state of each connector is evaluated with the aid of information about the topological connections among the connectors which are generated in the route-searching procedure. It is possible to determine, referring to the restrictions or requirements about the states of connectors given as the security conditions, the sequence of valve operations.

\section{Introduction}

It is evident that the goal of process control is to maintain the process accurately at the required conditions. In this respect, the technology of process control is considered to have an important role in the context of the safety administration of chemical processes. Feedback control has been widely adopted in chemical plants and is now the basis of the computer control systems including DDC or the production management system.

However, reflecting on the fact that so many accidents happened successively in chemical plants in Japan during the last couple of years, a control system is being called for that can deal with the problems of countereemergency or safety management in addition to the steady-state control modes. There is, of course, no doubt that such requirement can only be attained with the aid of computers.

It is quite essential to set up, as the philosophy of process design, a border between the functions of man

Received December 3, 1977 and machine in plant operations. In other words, it is not appropriate to replace any part of the heuristic responsibility by a mechanical function only because the machine can handle it. Otherwise, the work left for human operators would be of a type that they may not be happy to perform, because they would be forced to function as a part of the mechanical system. In addition to this problem, there arise many difficulties in managing and coordinating the whole plant if the control system is a random mixture of man-controlled. and machine-controlled processes.

The most important factor that should be taken into account in the system design of computer-aided operating systems is the matching of man and machine responsibilities, particularly in the tackling of emergency problems. The predictable defects or troubles for which the causal dependencies are clear can in general be dealt with by computers, since it is not difficult to convert the schemes of judgment of operators in reacting to such troubles into computer language or algorithms. It is, therefore, one of the most important roles for the operators to discover defects that the computer can hardly detect and to 